\title{
Review: patient reminder or recall systems improve immunisation rates
}

Szilagyi P, Vann J, Bordley C, et al. Interventions aimed at improving immunization rates. Cochrane Database Syst Rev 2002;(4):CD003941 (latest version 23 Aug 2002).

\section{QUESTION: Are patient reminder or recall systems effective in improving immunisation rates?}

\section{Data sources}

Studies were identified by searching Medline, EMBASE/ Excerpta Medica, PsycINFO, Sociological Abstracts, CAB Health Abstracts, and the EPOC register; scanning reference lists of relevant studies and reviews; reviewing abstracts and proceedings from scientific meetings; and contacting study collaborators.

\section{Study selection}

Studies were selected if they were randomised controlled trials (RCTs), controlled before and after studies, or interrupted time series studies published in English that evaluated reminder or recall interventions aimed at reminding patients of immunisation visits that were due (reminder) or overdue (recall).

\section{Data extraction}

Data were extracted on patient age (newborn to adult); delivery (letter, postcard, telephone, autodialer, or in person), specificity (generic or personal reminders), and number (1 time or multiple reminders); and vaccination schedule. Study quality was assessed (allocation concealment, blinding, follow up, reliable primary outcome measure, and protection against contamination). Outcomes were immunisation rates or the proportion of the target population that was up to date on recommended immunisations.

\section{Main results}

41 studies met the selection criteria. The interventions included letters (21 studies), postcards (8 studies), telephone calls (7 studies), autodialer (4 studies), postcard plus phone call (1 study), and tracking and outreach (2 studies). 7 studies also evaluated provider and patient reminders combined. 37 RCTs (38 comparisons: 15 in children and 23 in adults) were included in a meta-analysis. Patients who received the reminder or recall intervention were more likely to be immunised or up to date with immunisations than were patients who did not receive the intervention (table). The greatest improvement in immunisation rates was seen with person to person telephone reminders (odds ratio [OR] $5.52,95 \%$ CI 3.90 to 7.79 ). Reminder or recall systems were similarly effective in increasing immunisation rates in children and adults and were effective for most types of vaccine (general [OR 2.49, CI 1.83 to 3.38], childhood setting (academic, public health, or private); intervention
Abstract and appear in ACP Journal Club. influenza [OR 4.25, CI 2.1 to 8.6], and adult [age $\geq 65 \mathrm{y}$ ] influenza [OR 2.25, CI 1.45 to 3.50]). Single and multiple reminders were similarly effective.

\section{Conclusion}

Patient reminder or recall systems are effective in improving immunisation rates.

\section{COMIMENTARY}

Vaccines revolutionised the prevention of infectious disease in the 20th century. Among the greatest remaining challenges in dealing with vaccine preventable disease is developing effective strategies for implementing vaccine programmes.

The systematic review by Szilagyi et al emphasises the value of communicating with patients and indicates that practice-based strategies can increase immunisation rates. This evidence is consistent with the recommendation of the National Immunization Program of the Centers for Disease Control and Prevention for using reminder systems to increase immunisation rates.

Effectively using recall or reminder systems relies on the ability to monitor patients' immunisation status and activate the system when an immunisation is due. Immunisation registries, which are confidential, computerised databases allowing records linkage and interactive tracking of vaccine delivery and receipt, are efficient means of monitoring immunisation status. ${ }^{1}$ However, only about one quarter of children in the US were included in active immunisation registries in $2001,{ }^{2}$ which probably diminishes the optimal implementation and effectiveness of immunisation recall or reminder systems.

The results of this meta-analysis should be interpreted cautiously. Cumulative immunisation rates were only $27 \%$ in non-intervention groups and $42 \%$ in those receiving recalls or reminders. $42 \%$ is disappointing. Despite analysis by baseline immunisation rate, it remains unclear if recall or reminder systems are effective at optimising coverage in populations with lower rates of immunisation.

Overall immunisation rates and use of immunisation registries in the US fall short of the Healthy People 2010 goals. ${ }^{3}$ Additional information on the effectiveness of immunisation recall and reminder systems stratified by personal disease risk, use and type of immunisation registry enrolment, and other patient and provider factors would be welcome in order to use these systems in the best way.

Paul V Targonski, MD, PhD

Gregory A Poland, MD Mayo Clinic and Mayo Foundation Rochester, Minnesota, USA

Immunisation rates for reminder or recall intervention v no intervention (38 comparisons)*

\begin{tabular}{llll}
\multicolumn{2}{ll}{ Weighted event rates } & & \\
\cline { 1 - 2 } Intervention & No intervention & RBI $(95 \% \mathrm{Cl})$ & NNT (Cl) \\
\hline $42 \%$ & $27 \%$ & $79 \%(57$ to 104$)$ & $7(6$ to 10$)$ \\
\hline
\end{tabular}

*Abbreviations defined in glossary; RBI, NNT, and $\mathrm{Cl}$ calculated from data in article using a random effects model. Follow up ranged from 1 week to 2 years.
1 National Immunization Program. www.cdc.gov/nip/ publications/adultstrat.htm

Immunization registry use and progress-United States, 2001. MMWR Morb Mortal Wkly Rep 2002;51:53-6.

US Department of Health and Human Services. Healthy People 2010. 2nd ed. Washington, DC: US Government Printing Office, November 2000. www.healthypeople.gov/ Document/tableofcontents.htm\#volumel. 